\title{
Past partum period: a neglected area of care
}

\author{
Dr. Nadeem Zaidi, FCPS, FCARCSI, Consultant Anaesthetist, Furness General Hospital
}

\section{INTRODUCTION}

Pregnancy is marked with various adaptive physiological and anatomical changes. These enable the female body to accommodate and facilitate the growth of the foetus. The time phase following the delivery of baby and placenta is termed as post-partum or puerperium. This is a transition period where females adjust themselves both psychologically and physically for a new role. Also, during this time pregnancy related anatomical and physiological changes revert back to normal, moreover the post-partum period may be associated with some new set of problems.

The World Health Organisation (WHO) describes the post-partum phase as the most neglected period of care. According to WHO statistics most maternal and infant deaths occur in the first month after birth; approximately $50 \%$ of these maternal deaths occur within the first 24 hours after delivery. ${ }^{1}$ According to MBRRACE-UK (Mothers and Babies Reducing Risk through Audits and Confidential Enquiries across the UK) pregnancy remains very safe across the United Kingdom (UK). According to UK statistics for the year 2015-17, 209 women $(9.2$ $/ 100,000)$ died during or up to six weeks after pregnancy. ${ }^{2}$

In the following review, general principles of maternal care in puerperium will be discussed. More emphasis has been laid on certain common problems occurring during this phase. The aim is to refresh knowledge about the pathophysiological changes happening in puerperium so that better care is delivered to women in the post-partum period.

\section{POST-PARTUM: DEFINITION}

The six-week period starting from the delivery of baby and placenta constitute the post-partum period or puerperium. During this time maternal physiological changes revert back to the pre-pregnant state. This transition may not be a linear process as some body organs may take a longer time to resume the base line level. Management strategies and priorities are different for the first six-twelve hours after delivery and for the subsequent length of puerperium. ${ }^{3}$

\section{FIRST SIX-TWELVE HOURS AFTER DELIVERY}

The first six-twelve hours after delivery of the baby are marked with rapid physiological changes and potentially life-threatening situations. During this time the patient is monitored for bleeding (post-partum haemorrhage), amniotic fluid embolism, eclampsia and problems related to anaesthesia. After delivery of the placenta uterine bleeding stops primarily by two mechanisms. Primary haemostatic mechanism is due to the contraction of myometrial muscles which constricts the intra myometrial vessels. In addition, large vessels at the placental site thrombose (secondary haemostatic mechanism). On examination a soft uterus in the presence of vaginal bleeding suggest uterine atonia. By 24 hours after delivery the uterine fundus should be felt as non-tender, firm at the level of umbilicus. Normal shedding of blood and decidua from the uterus is termed as lochia rubra it is red to reddish brown in colour and lasts for three-five days after delivery. This post-delivery vaginal discharge then changes colour to pinkish brown and called lochia serosa; this last for twothree weeks. Finally, lochia turns to whitish to yellowish white in colour (lochia alba) and may last for the duration of puerperium. The cervix and vagina contract slowly but never to its nulliparous state. ${ }^{3,4}$

Early maternal issues are fatigue, musculoskeletal pain, shivering, perineal discomfort and dysuria; the magnitude depends upon the mode of delivery and duration of the second stage of labour. Some patients may also complain of crampy abdominal pain, these after pains occur due to uterine contractions which help to stop bleeding by compressing the blood vessels at the placental site. This phenomenon is more common in multiparous women and during breast feeding due to release of oxytocin. Breast engorgement or swelling can be noticed after the delivery, this is due to tissue oedema and milk accumulation.,

Apart from maternal haemodynamic monitoring the patients need examination and clinical observation for uterine atonia, excessive vaginal and any occult bleeding. The perineum should be examined for oedema, discharge and dehiscence. Patients requiring general anaesthesia or regional anaesthetic techniques to facilitate delivery may need extra monitoring regarding the residual effects of anaesthetics.

\section{SIX WEEKS OF PUERPERIUM}

During the six weeks following delivery the maternal body undergoes a slow period of physical and emotional recovery. The post-partum period or puerperium is heavily influenced by various traditions, taboos, beliefs and rituals. In many cultures around the world specific practices are observed to ensure quick maternal recovery and avoid future illnesses. The puerperium is marked with certain mild self-limiting concerns (wound infection, recurrent urinary tract infection, constipation and haemorrhoids) to some severe pathologies. According to MBRRACE-UK ${ }^{2}$ heart disease remains the leading cause of death, followed by thromboembolic episodes during pregnancy and within six weeks after delivery.

\section{Post-partum cardiomyopathy (PPCM)}

Post-partum cardiomyopathy (PPCM) is a form of dilated cardiomyopathy affecting primarily the left ventricle of the heart. Women become symptomatic either in the last trimester of pregnancy or up to five months after delivery. Contractility of the left ventricle is impaired (systolic dysfunction) and the patient presents with signs and symptoms of heart failure; these patients are also more prone for arrhythmias and thromboembolic episodes. PPCM can occur in any women during her reproductive years but certain factors increase its risk (multiple pregnancies, maternal diabetes, nutritional status, preeclampsia/eclampsia, hypertension advanced maternal age, history of cardiac conditions and African heritage). Aetiology of PPCM is still uncertain but most of the research is pointing towards autoimmune dysfunction, 
genetics, trace elements deficiencies and viral infection. ${ }^{6}$ Diagnosis is difficult as most of the symptoms (shortness of breath, tiredness, fatigue, and swollen legs) are present in the last trimester of pregnancy. Early onset of symptoms, their intensity and presence even in resting state need the cardiologist's opinion. ECG, x-ray chest, echocardiography and MRI of the heart are helpful to confirm diagnosis. Treatment options need individualisation and depend upon the signs and symptoms and stage of labour. Diuretics, beta blockers and angiotensin converting enzyme (ACE) inhibitors are the main pharmacological agents used in these patients. Further follow up and counselling for future pregnancies is very important even after full resolution of symptoms. ${ }^{7}$

\section{Post-partum venous thromboembolism}

The hypercoagulable state of pregnancy predisposes the mother at increased risk for venous thromboembolic (VTE) episodes, with the puerperium being the most vulnerable phase in this regard. The relative risk of VTE is 60 - fold higher in women during puerperium when compared with the non-pregnant state. This reaches its peak at three-six weeks after delivery and then slowly subsides over a period of ten-twelve weeks post-partum. ${ }^{8}$ VTE can be prevented by careful evaluation of maternal risk factors during ante natal visits and by using appropriate pharmacological interventions after the delivery. Strongly correlated risk factors in this regard are advanced maternal age, caesarean section, preeclampsia, obesity, thrombophilia, lupus, postpartum haemorrhage, maternal systemic infection, preexisting cardiovascular diseases and in vitro fertilization. ${ }^{9}$ Low molecular weight heparin (LMWH) is recommended for acute treatment of VTE as well as thromboprophylaxis during pregnancy and in the post-partum phase.

\section{Post-partum neuropathies}

Pregnancy is also associated with various neuropathies; these are generally in the form of mononeuropathies, plexopathy, radiculopathy and poly neuropathies. The most common peripheral neuropathies associated with the pregnancy and post-partum period are Bell's palsy, carpel tunnel syndrome and lower limb neuropathies. Pathophysiology of most of them is related to the pregnancy and various hormonal changes associated with it. ${ }^{10}$ The majority of the lower limb peripheral neuropathies noticed in the postpartum period are often termed as intrinsic obstetrical palsies. Maternal malpositioning during labour, prolonged second stage of labour, instrumental delivery and cephalo pelvic disproportion causes mechanical trauma (stretch, compression) to the peripheral nerves of the lower limbs at vulnerable anatomical points which produces a mixture of sensory motor deficits. Femoral and lateral cutaneous nerves of the thigh are the most commonly involved nerves. A small number of lower limb neuropathies are often related to various neuraxial anaesthetic techniques, used for labour analgesia or to facilitate the process of delivery. ${ }^{11}$

\section{Post-partum mental health issues}

Approximately 15\% of women experience some sort of mental health issues in their early motherhood phase.
The spectrum of these psychiatric disorders is quite wide and varies from mood swings to post-natal depression, anxiety, psychosis and labour related post-traumatic stress disorders. Risk factors in this regard are pre-pregnancy stress, low socioeconomic support, teenage pregnancy and child birth related issues (fear of childbirth, difficult and prolonged labour) and a history of pre-existing psychiatric disorders. ${ }^{12}$ According to MBRRACE - UK statistics maternal suicide is the fifth most common cause of death during pregnancy and immediately afterwards, but is the leading cause of death after the first year of pregnancy. ${ }^{2}$

Approximately $85 \%$ of women have some sort of mood changes and swings in the puerperium (baby blues). These symptoms are mild, self-limiting and peak in the first four-five days after delivery. High hormonal fluctuations following childbirth are being implicated toward its aetiology. Baby blues does not require any treatment, but persistence of symptoms may need expert intervention. About $10-20 \%$ of new mothers suffer from major depression following the birth of the baby (postpartum depression -PPD). Symptoms of PPD (loss of appetite, anxiety, irritability, sleep disturbances, panic attacks and severe mood swings) may last up to a year. Antidepressant medications and counselling of mothers during the puerperium and the subsequent pregnancy are the main treatment modalities available in this regard.

Postpartum psychosis (PPP) affects less than $1 \%$ of new mothers. Onset of symptoms is generally within the first couple of weeks following child birth. Confusion, irritability, memory loss, delusions, hallucinations and preoccupation with trivial acts are the main symptoms. Patients may need hospitalisation to control the symptoms. Mothers who suffer from postpartum psychosis are highly likely to manifest its symptoms in subsequent pregnancies. ${ }^{13}$

\section{Post-partum endocrinal issues}

Post-partum thyroiditis affects about 5\% of mothers. Pregnancy is associated with immunosuppression, resulting in the formation of anti-thyroid antibodies, these antibodies produce thyroid dysfunction in two distinct phases. The initial phase is hyperthyroidism which lasts for two-six months after delivery. This is followed by a hypothyroid state which can occur between three-twelve months post-partum. At one-year post-partum the thyroid gland assumes a euthyroid state in more than $95 \%$ of women. ${ }^{14}$

About $50 \%$ of gestational weight gain is lost in the first six months after delivery. The remainder of the gestational weight gain is slowly lost over the over the next six months. Most women gain weight after the delivery, mean weight gain at six months post-partum is about 4.5 $-5.5 \mathrm{~kg}$. Various risk factors for excessive weight retention in the post-partum phase are pre-pregnancy high BMI, more weight gain in the pregnancy, ethnicity, maternal age (adolescents), pregnancy intervals, life style and activity in the post-partum phase. ${ }^{15}$

Post-partum alopecia occurs in some women due to the rapid fall in oestrogen levels in the body. It reaches its peak by four months after delivery but hair growth pattern assumes its normal pattern by one-year past partum. ${ }^{16}$ 


\section{SIX WEEKS AFTER DELIVERY}

Most of the problems and complications which happen in pregnancy and labour slowly settle down between six weeks and six months after delivery and any new pathology is rare. Abdominal and perineal muscles regain most of their prepregnant tone. Separation of rectus abdominus (diastasis) may persist in some patients. As the infant's night time sleep increases, the mother's night time resting improves also; this has a positive impact on her mood and physical health.

\section{POST-PARTUM FOLLOW-UP}

The routine post-partum visit should occur at four to six weeks after vaginal delivery and at one to two weeks after caesarean section. Frequency, timing of these visits, sites (home, hospital, telephonic discussion), to be seen by GP, community midwife or hospital consultant depends upon the individual need. These visits provide an opportunity for maternal support, counselling and referrals. Maternal support and education are required for breast feeding, self-care, pelvic floor exercises, level of activity, nutrition, diet, contraception and subsequent pregnancies. The mother and infant wellbeing are assessed and any concerns raised by mother should be addressed during these visits. Any morbidity, if happened during pregnancy and labour, should be investigated in the post-partum phase in terms of its aetiology, risk of recurrence and prevention. Appropriate referrals if required should also be made during these visits.

\section{REFERENCES}

1. World Health Organisation. WHO recommendations on postnatal care of the mother and newborn. Geneva: WHO; 2013.

2. MBRRACE-UK: mothers and babies: reducing risk through audits and confidential enquiries across the UK. Available at: https://www.npeu.ox.ac.uk/mbrrace-uk (accessed 09.03.2020).

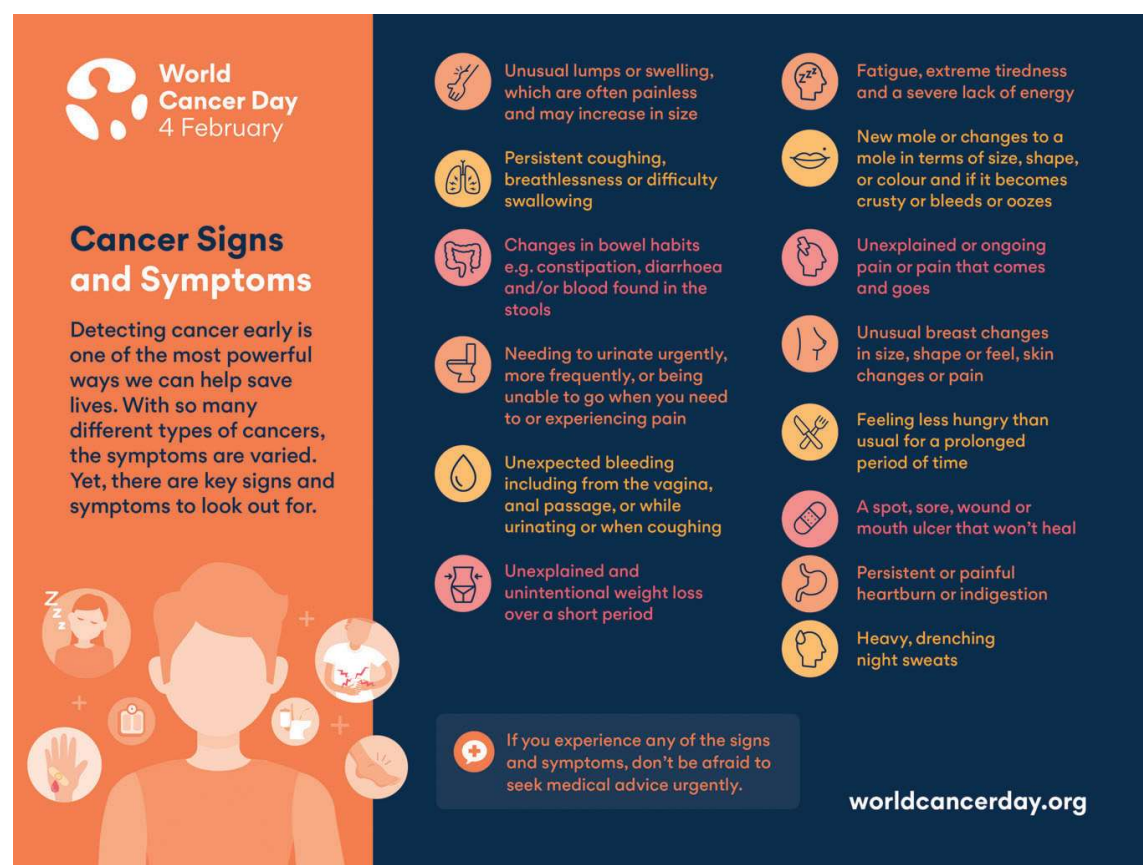

3. Mattea R, Alessandra C, Beatrice LR. Post-partum period: three distinct but continuous phases J Prenat Med 2010;4(2):22-25.

4. Berens, P. Overview of the postpartum period: Physiology, complications, and maternal care. Available at: www. uptodate.com (accessed 09/03/2020).

5. American College of Obstetricians \& Gynaecologists. Committee on Obstetric Practice. Committee Opinion No 736 Optimizing postpartum care. Obstet Gynecol. 2018;131(5):e140-150.

6. Pramesweri HS, Dewi TI, Hasan M, Martanto E, Aprami TM. Hypertension in pregnancy as the most influential risk factor for PPCM. Br J Cardiol. 2018; 25:111-114.

7. Hilfiker-Kleiner D, Haghikia A, Nonhoff J, Bauersachs J. Peripartum cardiomyopathy: current management and future perspectives. Eur Heart J. 2015; 36(18):1090-1097.

8. Hellgren $M$, Mistafa O. Obstetric venous thromboembolism: a systematic review of dalteparin and pregnancy. J Obstet Gynaecol. 2019;39(4):439-450.

9. Tepper NK, Boulet SL, Whiteman MK, Monsour M, Marchbanks PA, Hooper WC, et al. Postpartum venous thromboembolism: incidence and risk factors. Obstet Gynecol. 2014;123(5):987-996.

10. Klein A. Peripheral nerve disease in pregnancy. Clin Obstet Gynecol. 2013;56(2):382-88.

11. O'Neal MA, Chang LY, Salajegheh MK. Postpartum spinal cord, root, plexus and peripheral nerve injuries involving the lower extremities: a practical approach. Anesth \& Analg. 2015;120 (1):141-48.

12. O'Hare MW, Wisner KL. Perinatal mental illness: definition, description and aetiology. Best Pract Res Clin Obstet Gynaecol. 2014;28(1):3-12

13. O'Hara MW, McCabe JE. Postpartum depression: current status and future directions. Annu Rev ClinPsychol. 2013;9:379-407.

14. Smith A, Eccles-Smith J, D'Emden M, Lust, K. Thyroid disorders in pregnancy and postpartum. Aust Prescr. 2017;40(6):214-219.

15. Endres LK, Straub H, McKinney C, Plunkett B, Minkovitz $\mathrm{CS}$, Schetter CD. et al. Postpartum weight retention risk factors and relationship to obesity at 1 year. Obstet Gynecol. 2015;125(1):144-152.

16. Eastham JH. Postpartum alopecia. Ann Pharmacother 2001;35(2):255-258.

Correspondence to: syed-nadeem.zaidi@mbht.nhs.uk

New Faster Diagnosis Standard for cancer are being introduced across the country in Aprill 2020 